\title{
Optical Transient Surveys
}

\author{
Brian Schmidt \\ Research School of Astronomy and Astrophysics, Australian National University, \\ Weston Creek, ACT 2611, Australia \\ email: brian@mso.anu.edu.au
}

Invited Talk

\begin{abstract}
The last five years have seen an explosion of activity to monitor the sky at optical wavelengths. The following summarizes an overview of the range of experiments currently being, or soon to be, undertaken in both cataloguing and monitoring the sky, and suggests scientific opportunities for the short- to medium- term in the arena of optical transient astronomy. In so doing, it applies the philosophy described by Warner (page 3) to the gamut of variability studies that are now burgeoning in observational astronomy.
\end{abstract}

\section{History}

Studies of transient objects have played a key role in the historical development of Astronomy. Henrietta Swan Leavitt's discovery of the Cepheid period-luminosity relationship, and its use to set the scale of the universe, is an early example. The first studies of extra-galactic transients, such as supernovæ, were empowered by dedicated hardware such as the 18-inch Schmidt telescope used by Zwicky to scan the night skies for supernovæ by "blinking" large photographic plates. This was the same technique used 50 years later by the Calan-Tololo SN search to define the set of Type Ia supernovæ which paved the way for discovering the accelerating universe. Over the past 20 years, a new set of optical survey telescopes, powered by increasingly large digital CCD detectors, are revolutionising the field.

\section{Methodology}

Transient detection is typically done in two ways. One method is through the comparison with a catalogue of objects, where variability is monitored and new objects catalogued. This is computationally less demanding, is good where very high precision is required, but results in poor detection efficiency near the detection threshold, or in crowded regions. The other method - image subtraction - where images are matched to a template and the template subtracted, is more computationally demanding and has poorer absolute precision, but leads to much better transient detection efficiency across a survey.

\section{Science Drivers}

Currently, there is a range of science themes that drive transient surveys. They include transits, where planets are identified by their transits in front of their host stars, gravitational microlensing, which is used to undertake novel studies of stars and planets, and to measure the stellar column densities towards the Milky Way and other nearby galaxies, discoveries of asteroids, to quantify the dynamics of the solar system and to look for potential Earth-colliding bodies, variable stars, which are used to understand 
the physics of stars and stellar systems, extragalactic transients, which are used to explore the violent physical processes involved in stellar explosions, black hole accretion and the unknown, and cosmology, where transient objects are used to probe the scale and content of the universe.

\section{Figure of Merit}

The figure of merit for a transient search, the etendue, depends precisely on the objectives of a survey, but can generically be described as the rate a search can observe objects to a fixed brightness limit. In the sky-limited case, it is interesting to note that it was not until the commissioning of the MACHO Camera on the Great Melbourne Telescope in 1992 that a digital system exceeded the etendue of the photographic Schmidt telescopes. The state-of-the art etendue currently resides with the CFHT+MegaCam instrument, but will soon eclipsed by the CTIO-4m+DECCAM, by the Subaru+Hyper Suprime Cam, and eventually, by LSST. These instruments will be 150-2200 times quicker at surveying the sky than was the 48-inch Schmidt Telescope. But many transient science cases are not about just going deep quickly. Planetary transits and nearby supernova searches are cases in point, where high precision and large areal coverage are the key factors. Ultimately, the success of a project depends on its execution with respect to its scientific goals - good execution can make up for a lot of etendue. 